\title{
Pilot Study: An Ocular Biomarker for Diagnosis of Attention Deficit Hyperactivity Disorder
}

\author{
Sujin $\mathrm{Bae}^{1}$, Jee Taek $\mathrm{Kim}^{2}$, Jung Min $\mathrm{Han}^{3}$, and Doug Hyun $\mathrm{Han}^{4} \bowtie$ \\ ${ }^{1}$ Office of Research, Chung-Ang University, Seoul, Republic of Korea \\ ${ }^{2}$ Department of Ophthalmology, College of Medicine, Chung-Ang University, Seoul, Republic of Korea \\ ${ }^{3}$ Industry Academic Cooperation Foundation, Chung-Ang University, Seoul, Republic of Korea \\ ${ }^{4}$ Department of Psychiatry, College of Medicine, Chung-Ang University, Seoul, Republic of Korea
}

Objective Biomarkers of attention deficit hyperactivity disorder (ADHD) are crucial for early diagnosis and intervention, in which the identification of biomarkers in other areas of the body that represent the immature brain of children with ADHD is necessary. The present study aimed to find biomarkers of ADHD in the retina and assessed the relationship between macular thickness of the retina and cortical thickness of the brain in children with ADHD.

Methods Twelve children with ADHD and 13 control children were recruited for the study. To find ocular markers of ADHD, we investigated the correlation between clinical symptoms of ADHD assessed with the Korean ADHD Rating Scale (K-ARS), cortical thickness of the brain, and macular thickness measured with the mean thickness from the Early Treatment Diabetic Retinopathy Study (ETDRS).

Results Children with ADHD showed increased macular thicknesses quantified as an ETDRS ring in both eyes, compared to control subjects. Moreover, the right inner ETDRS ring had a positive correlation with K-ARS scores. The ADHD group had an increased ratio of thickness of the right frontal lobe to that of the parietal cortex, compared with the control group. There were positive correlations between the means of the inner ETDRS ring (right) and the left paracentral/right isthmus cingulate thicknesses in the control group. However, there were negative correlations between the means of the inner ETDRS ring (right) and the left frontal pole/right pars triangularis thicknesses in the ADHD group. The results of both groups were at the uncorrected level.

Conclusion The different patterns of macular thickness might represent the immature cortical thickness of the brain in children with ADHD.

Psychiatry Investig 2019;16(5):370-378

Key Words Attention deficit hyperactivity disorder, Brain, Magnetic resonance imaging, Biomarker, Macular thickness.

\section{INTRODUCTION}

Attention deficit hyperactivity disorder (ADHD) has been considered a neurodevelopmental disorder with a prevalence of $2.5^{1}-7.2 \% .^{2}$ Despite its high prevalence, controversy remains with respect to its diagnosis due to the lack of biological evidence. ${ }^{3}$ In addition, misdiagnosis of ADHD can impede early intervention and overdiagnosis may lead to unnecessary use of medication for treatment. Many clinicians and researchers continue to search for biological markers to guide the di-

\footnotetext{
Received: May 31, 2018 Revised: October 9, 2018

Accepted: February 26, 2019

$\triangle$ Correspondence: Doug Hyun Han, MD, PhD

Department of Psychiatry, College of Medicine, Chung-Ang University, 84 Heukseok-ro, Dongjak-gu, Seoul 06974, Republic of Korea Tel: +82-2-6299-3132, Fax: +82-2-6298-1508, E-mail: hduk70@gmail.com

(a) This is an Open Access article distributed under the terms of the Creative Commons Attribution Non-Commercial License (https://creativecommons.org/licenses/bync/4.0) which permits unrestricted non-commercial use, distribution, and reproduction in any medium, provided the original work is properly cited.
}

agnosis of ADHD.

Numerous brain imaging studies have highlighted a cortical developmental lag in children with ADHD.,5 In a longitudinal study, the prefrontal cortex and middle/superior temporal cortex were the pronounced regions in which maturational delays were found in the $\mathrm{ADHD}$ group compared to the healthy group. ${ }^{4}$ Many other studies using cross-sectional designs have also found that participants with ADHD showed decreased cortical thickness in the frontal, ${ }^{6,7}$ parietal, ${ }^{7}$ and temporal cortices. ${ }^{8}$ Several neuroimaging study groups have recently taken interest in patterns of brain asymmetry in ADHD. ${ }^{9}$ In a longitudinal study, left-right cortical asymmetry was found in the frontal cortex of children with ADHD, unlike in control group. ${ }^{9}$ The authors suggested that the absence of a pattern of asymmetry in the frontal region in the ADHD group might imply abnormal development of the prefrontal cortex. ${ }^{9}$ The healthy youth in this study showed greater cortical thickness in the right frontal and left occipital lobes with aging, exhibiting an 
association between anatomical asymmetry in the brain and typical lateralization of cognitive function. ${ }^{9}$

As reported above, brain imaging studies have supported the existence of biological markers of ADHD in children. However, these studies are limited in their applicability for the study of children in terms of their low accessibility due to the intimidating environment of magnetic resonance scanners. ${ }^{10}$ To overcome this obstacle, peripheral biomarkers such as macular thickness promise to open a new avenue for investigating the developing brain. The major advantages of a methodology employing macular thickness have been described in a recently published article, and includes the similarity between the brain and retina from an embryological perspective and the relatively simple accessibility of retinal measures compared to brain scans. ${ }^{11}$ In recent decades in psychiatry, the retina has been considered to represent the brain status, suggesting the suitability of conducting retinal studies to investigate schizophrenia and Alzheimer's disease (AD). Decreases in the retinal nerve fiber layer (RNFL) and macular thickness have been found in a schizophrenia cohort in recent a study, ${ }^{12}$ and in patients with $\mathrm{AD}$, a significant reduction in RNFL thickness was also found. ${ }^{13}$ Moreover, Iseri et al have demonstrated a relationship between retinal pathology and cognitive impairment in an $\mathrm{AD}$ group. ${ }^{14} \mathrm{As}$ for $\mathrm{ADHD}$, Hergüner reported lower RNFL thickness, but no difference in macular thickness. ${ }^{15}$

Based on the aforementioned background, we conducted this study to investigate the possible diagnostic utility of the retina in children with ADHD. We hypothesized that children with ADHD would show a significant difference in the ratio of their brain cortices and macular thickness than healthy control children, and that an alteration in macular thickness might be associated with altered brain cortical thickness.

\section{METHODS}

\section{Participants}

Twelve children with ADHD and 13 healthy control children were recruited from the Department of Psychiatry of Chung-Ang University Hospital in Seoul, Korea. The procedures were explained to the participants and informed consent was obtained from the children and their parents. The study protocol was approved by the Institutional Review Board of Chung-Ang University Hospital (C2012033). This research followed the tenets of the Declaration of Helsinki.

All participants were assessed with the Korean Kiddie Schedule for Affective Disorders and Schizophrenia-Present and Lifetime version ${ }^{16}$ and confirmed by a child and adolescent psychiatrist through clinical interviews. All children with ADHD completed the Children's Depression Inventory $(\mathrm{CDI})^{17}$ to detect the presence of depressive symptoms and their parents completed the Korean ADHD rating scale (K-ARS) for patients. ${ }^{18}$ Finally, intelligence quotient (IQ) was assessed by Korean-Wechsler Intelligence Scale for Children.

The exclusion criteria were a history of an axis I psychiatric disorder other than ADHD, taking psychiatric medications, IQ below 80 , a history of neurological or medical disorders, claustrophobia, any ocular condition that could affect vision, a history of retinopathy of prematurity or amblyopia, best corrected visual acuity (BCVA) below 1.0, myopic eyes under -6.0 diopter, and hyperopic eyes over 6.0 diopter.

\section{Macular thickness measurement}

All children in this study underwent full ophthalmologic examinations including cycloplegic refraction, BCVA measurement, slit-lamp biomicroscopy, and detailed fundus examination. Visual fields evaluated the participant's concentration using a Humphrey Field Analyzer (HFA II; Carl Zeiss Meditec, Inc.; Dublin, CA, USA) with the 24-2 SITA Fast program. Reliability indices, including fixation losses, false positives, false negatives, and mean deviation (MD) of the visual field test were presented. Fundus photographs were captured in both eyes with a Topcon TRC-NW7SF (Topcon; Tokyo, Japan). We obtained nine values of macular thickness for all participants according to the Early Treatment Diabetic Retinopathy Study (ETDRS) grid. ${ }^{19}$ We compared the differences in three values (central subfield, mean of inner ETDRS ring, and mean of outer ETDRS ring) to explore global differences rather than minor alterations. Measurements of macular thickness were acquired for both eyes using the Cirrus OCT Model 4000 (Carl Zeiss Meditech; Jena, Germany).

\section{Magnetic resonance imaging acquisition}

All participants underwent brain magnetic resonance imaging (MRI) with a 3.0 T Philips MRI scanner (Philips; Eindhoven, the Netherlands). Three-dimensional T1-weighted images were acquired using a Turbo Field Echo sequence with the following parameters: 180 sagittal slices, repetition time $=$ $8.0-8.4 \mathrm{~ms}$, echo time $=3.6-4.0 \mathrm{~ms}$, field of view $=220 \times 220$ $\mathrm{mm}$, flip angle $=8^{\circ}, 256 \times 256$ matrix, $1 \times 0.8594 \times 0.8594 \mathrm{~mm}^{3}$ voxel size. Each T1-weighted image was visually inspected before analysis to ensure that there were no artefacts or severe abnormalities that might affect further processing.

\section{Imaging data analysis}

Cortical reconstruction was performed using FreeSurfer software (version 5.3.0). All procedures were performed fully automatically, and a representative outcome is depicted in Figure 1. This processing included motion correction and averaging $^{20}$ of each participant's T1-weighted images, exclusion of non-brain tissue using a hybrid watershed/surface deforma- 

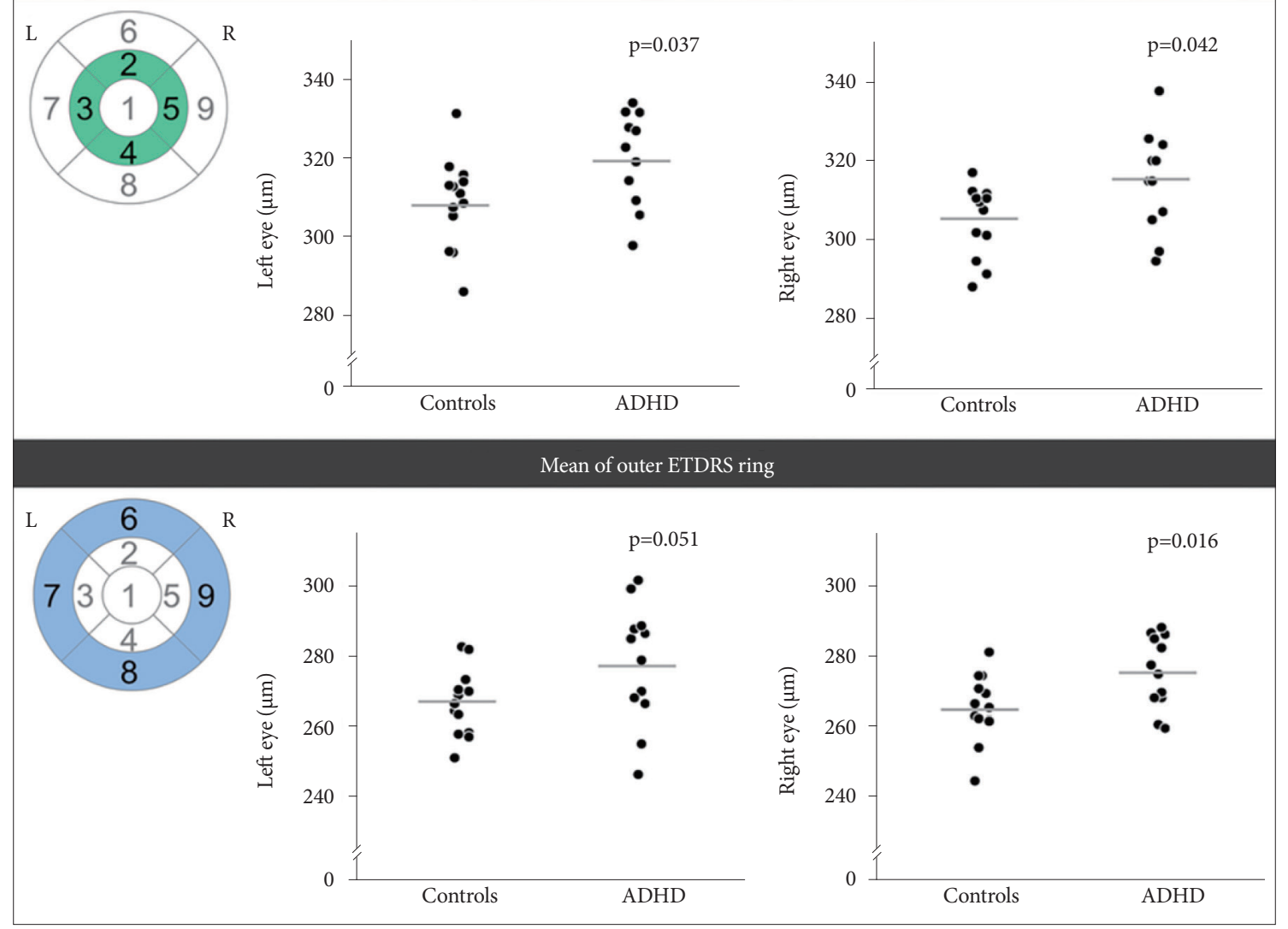

Figure 1. Macular thickness differences between healthy comparison children and children with ADHD. The independent t-test was used for group comparisons. Multiple comparison issues were not considered for the purpose of exploratory analysis. The gray lines represent the means of each group. ETDRS: Early Treatment Diabetic Retinopathy Study, ADHD: attention deficit hyperactivity disorder.

tion procedure, ${ }^{21}$ automated Talairach transformation, ${ }^{22}$ intensity normalization, ${ }^{23}$ tessellation of the gray matter/white matter boundary, automated topology correction, ${ }^{24}$ and surface deformation following intensity gradients to optimally position the gray/white and gray/cerebrospinal fluid borders. ${ }^{25,26}$ After completion of this process, a set of deformable procedures were performed, including surface inflation, ${ }^{27}$ registration to a spherical atlas, ${ }^{28}$ parcellation of the cerebral cortex into units, ${ }^{29}$ and creation of various surface-based data. This method utilizes both intensity and continuity information from the entire 3D MR image in segmentation and deformation processes to create representations of cortical thickness. ${ }^{26}$ Mean thicknesses of the frontal, parietal, temporal, and occipital cortices were acquired from the labels consisting of all relevant regions of interest (ROIs) in the Desikan-Killiany atlas. Procedures to obtain the outcomes of cortical thickness have been validated in post-mortem and in vivo studies, ${ }^{30}$ as well as a manual segmentation approach. ${ }^{31}$

\section{Statistical analyses}

All statistical analyses were conducted using SPSS version
21 (IBM Corp.; Armonk, NY, USA). We compared differences in age and IQ between groups by Mann-Whitney $\mathrm{U}$ test $(\mathrm{p}<$ 0.05 ). The differences in each group's macular thicknesses were analyzed via independent $t$-test $(\mathrm{p}<0.05)$. The Bonferroni correction method was implemented for multiple comparisons to reduce false positive results in all analyses that included the brain ( $\mathrm{p}<0.0125$, for four comparisons) and macula ( $\mathrm{p}<0.008$, for six comparisons). The relationship between K-ARS scores and macular thicknesses extracted from the ROIs in the group analysis was analyzed using the Pearson correlation test in the ADHD group.

Group differences in the ratios of frontal to parietal cortex, frontal to temporal cortex, and temporal to parietal cortex thickness were examined by independent $\mathrm{t}$-test $(\mathrm{p}<0.05)$. The ratio of frontal to parietal cortex thickness was calculated using the following formula: (mean cortical thickness of frontal cortex/mean cortical thickness of parietal cortex $\times 100$. The ratios of frontal to temporal cortex and temporal to parietal cortex thickness were acquired in the same manner.

The Pearson correlation test was used to examine the relationship between cortical and macular thickness. We used 34 
parcellated regions obtained from FreeSurfer's processing pipeline and the macular area extracted from the previous correlation analysis in the $\mathrm{ADHD}$ group.

\section{RESULTS}

\section{Demographics and clinical characteristics}

The age ranges in the control and ADHD groups were not significantly different $(9.8 \pm 2.1$ and $10.6 \pm 1.9$, respectively). The IQs of the healthy control children were higher than those of the children with ADHD; the ADHD group had higher K-ARS scores than the control group, and CDI scores did not differ between the groups (Table 1).

\section{Differences in macular thickness}

The mean of the inner ETDRS ring thickness (left eye) was thinner in controls $(308.8 \pm 11.4 \mu \mathrm{m})$ than in children with $\mathrm{ADHD}$ (319.1 $\pm 11.9 \mu \mathrm{m} ; \mathrm{p}=0.04)$. Moreover, the right eye (mean of inner ETDRS ring) showed increased thicknesses in children with $\mathrm{ADHD}(315.0 \pm 12.4 \mu \mathrm{m})$ relative to controls $(305.6 \pm 9.5$ $\mu \mathrm{m} ; \mathrm{p}=0.04)$. The ADHD group had higher values than the control group for means of the outer ETDRS ring thickness [277.8 $\pm 17.0 \mu \mathrm{m}$ and $266.6 \pm 9.5 \mu \mathrm{m}$, respectively, for the left eye; $275.6 \pm 10.3 \mu \mathrm{m}$ and $265.4 \pm 9.5 \mu \mathrm{m}$ for the right eye, re- spectively $(\mathrm{p}=0.02)]$. However, none of these outcomes remained significant after Bonferroni correction. The central subfield of the bilateral eyes did not differ between the groups. These results are presented in Table 2 and Figure 1.

\section{Relationship between macular thickness and K-ARS scores}

We found that the inner right ETDRS ring thickness had a positive correlation with K-ARS scores $(r=0.66$; $\mathrm{p}=0.036)$ (Figure 2) in the ADHD group. The ETDRS ring thickness of left inner $(\mathrm{r}=0.46 ; \mathrm{p}=0.183)$, right outer $(\mathrm{r}=0.24 ; \mathrm{p}=0.500)$, and left outer $(\mathrm{r}=-0.01 ; \mathrm{p}=0.977)$ had no correlation with K-ARS scores.

\section{Cortical thickness analysis}

Whole brain analysis with cluster-wise correction for multiple comparisons presented no significant difference in cortical thickness between the groups. There were no differences in the mean thicknesses of the frontal, parietal, temporal, or occipital cortex between the control and ADHD children. However, the ADHD group had an increased ratio of right frontal to parietal cortex thickness $(114.7 \pm 3.3)$ compared to the healthy comparison group (112.2 $\pm 2.2 ; \mathrm{p}=0.04)$ (Table 3$)$. However, none of these results remained significant after Bonferroni cor-

Table 1. Demographics and characteristics of the study participants

\begin{tabular}{|c|c|c|c|}
\hline \multirow{2}{*}{ Variable } & \multicolumn{2}{|c|}{ Mean (SD) } & \multirow{2}{*}{$\mathrm{p}^{*}$} \\
\hline & Controls & ADHD & \\
\hline \multicolumn{4}{|l|}{ Demographics } \\
\hline Sample size & 13 & 12 & - \\
\hline Age (years) & $9.8(2.1)$ & $10.6(1.9)$ & 0.33 \\
\hline Age (range, years) & $7-13$ & $7-13$ & - \\
\hline Sex (male/female) & $11 / 2$ & $8 / 4$ & $0.30^{\dagger}$ \\
\hline IQ & $102.8(9.4)$ & $91.0(16.4)$ & 0.04 \\
\hline \multicolumn{4}{|l|}{ Characteristics } \\
\hline \multicolumn{4}{|l|}{ Best corrected visual acuity } \\
\hline Left eye & $1.0(0)$ & $1.0(0)$ & 1.0 \\
\hline Right eye & $1.0(0)$ & $1.0(0)$ & 1.0 \\
\hline \multicolumn{4}{|c|}{ Intraocular pressure $(\mathrm{mm} \mathrm{Hg})$} \\
\hline Left eye & $15.7(3.1)$ & $16.3(3.0)$ & 0.68 \\
\hline Right eye & $15.8(2.9)$ & $16.9(2.1)$ & 0.48 \\
\hline \multicolumn{4}{|c|}{ Refractive errors, SE (diopter) } \\
\hline Left eye & $-2.0(1.6)$ & $-1.2(2.2)$ & 0.15 \\
\hline Right eye & $-1.9(1.7)$ & $-1.5(2.1)$ & 0.50 \\
\hline K-ARS & $5.7(3.8)$ & $18.5(15.9)$ & 0.04 \\
\hline CDI & $9.8(10.2)$ & $10.6(9.4)$ & 0.69 \\
\hline
\end{tabular}

*the t-test was used for group comparisons, the chi-square test was used for group comparisons. SE: spherical equivalent, K-ARS: Korean attention deficit hyperactivity disorder (ADHD) rating scale, CDI: Children's Depression Inventory, SD: standard deviation 
Table 2. Group differences of the macular thickness

\begin{tabular}{|c|c|c|c|c|}
\hline \multirow{2}{*}{ Region } & \multicolumn{2}{|c|}{ Mean (SD) } & \multirow{2}{*}{ Differences $(95 \% \mathrm{CI})$} & \multirow{2}{*}{$\mathrm{p}$} \\
\hline & Controls $(\mathrm{N}=13)$ & $\operatorname{ADHD}(\mathrm{N}=12)$ & & \\
\hline Central subfield (left) & $249.3(20.4)$ & $252.0(22.2)$ & $-2.7(-20.3$ to 14.9$)$ & 0.76 \\
\hline Central subfield (right) & $246.0(20.3)$ & $246.5(18.3)$ & $-0.5(-16.5$ to 15.5$)$ & 0.95 \\
\hline Mean of inner ETDRS ring (left) & $308.8(11.4)$ & $319.1(11.9)$ & $-10.3(-19.9$ to -0.7$)$ & 0.04 \\
\hline Mean of inner ETDRS ring (right) & $305.6(9.5)$ & $315.0(12.4)$ & $-9.5(-18.6$ to -0.4$)$ & 0.04 \\
\hline Mean of outer ETDRS ring (left) & $266.6(9.5)$ & $277.8(17.0)$ & $-11.2(-22.5$ to 0.1$)$ & 0.05 \\
\hline Mean of outer ETDRS ring (right) & $265.4(9.5)$ & $275.6(10.3)$ & $-10.3(-18.4$ to -2.1$)$ & 0.02 \\
\hline
\end{tabular}

The independent t-test was used for group comparisons. The digits of all the values are $\mu \mathrm{m}$. ADHD: attention deficit hyperactivity disorder, ETDRS: Early Treatment Diabetic Retinopathy Study, SD: standard deviation, CI: confidence interval

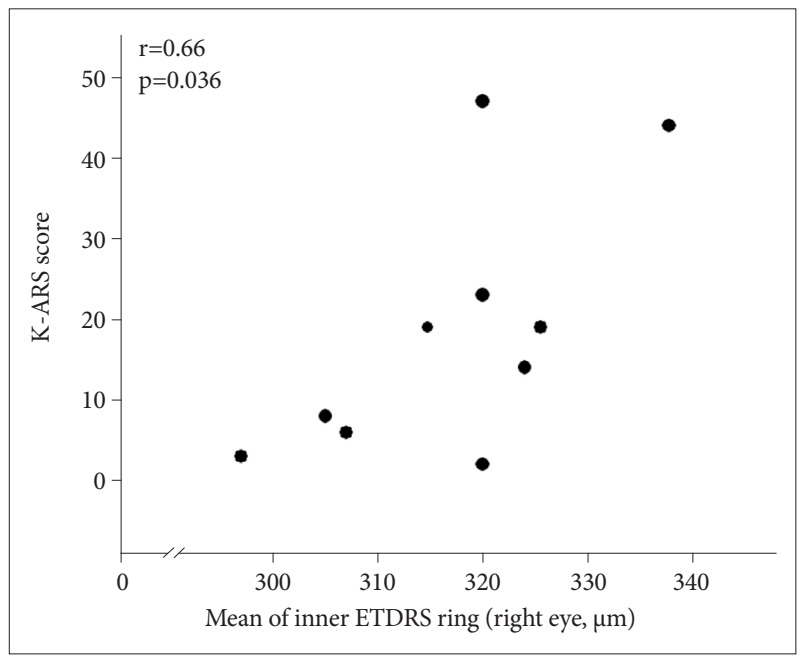

Figure 2. Relationship between macular thickness and K-ARS scores in the ADHD group. Pearson correlation test was used for the analysis. All levels of significance were set at 0.05 . K-ARS: Korean ADHD Rating Scale for Patients, ADHD: attention deficit hyperactivity disorder.

rection. The other comparisons, between the frontal and parietal cortices (left hemisphere) and between the frontal and temporal cortices (bilateral hemisphere), revealed no significant differences.

\section{Relationship between macular and cortical thickness}

Correlation analysis revealed that the mean of the inner ETDRS ring thickness was related to the cortical thickness of two brain clusters in children with ADHD and two alternate brain clusters in healthy control children. These results survived after correction for multiple comparisons using Bonferroni correction $[\mathrm{p}<0.0015(0.05 / 34)$, considering 34 ROIs derived from the Desikan-Killiany atlas]. The mean of the inner right ETDRS ring thickness was positively correlated with thickness of the left paracentral thickness $(r=0.81 ; p=0.0008)$ and the right isthmus cingulate $(\mathrm{r}=0.80 ; \mathrm{p}=0.0010)$ in the control group (Figure 3).

The mean of the inner right ETDRS ring thickness was negatively associated with left frontal pole $(\mathrm{r}=-0.82 ; \mathrm{p}=0.0009)$ and right pars triangularis $(\mathrm{r}=-0.82 ; \mathrm{p}=0.0011)$ thickness in the ADHD group (Figure 4). The other correlation analyses regarding the mean of the inner left ETDRS ring thickness or the mean of the outer bilateral ETDRS ring thickness yielded no significant differences after Bonferroni correction.

\section{DISCUSSION}

We found that children with ADHD had enlarged macular thickness compared to healthy control children. The inner ETDRS ring thickness of each participant in the ADHD group's right eye had a positive correlation with the K-ARS scores. In addition, children with ADHD had an increased ratio of thickness of the right frontal to the parietal cortex compared to healthy control children. We also observed positive correlations between the mean of the inner right ETDRS ring and left paracentral/right isthmus cingulate thickness in the control group. However, in the ADHD group, there were negative correlations between the mean of the inner right ETDRS ring and left frontal pole thickness, as well as right pars triangularis thickness.

In the present study, macular thickness increased bilaterally in children with ADHD relative to healthy controls, and these enlarged macular aspects were significantly correlated with several regions of the frontal and parietal cortices. To our knowledge, few studies have investigated macular thickness in children with ADHD. ${ }^{15}$ Herguner et al. ${ }^{15}$ suggested no difference in the thickness of the retina between patients with ADHD and healthy control subjects. Herguner et al. ${ }^{15}$ found no differences in macular thickness between the ADHD and control groups. The discordance between Herguner's results and ours might come from the different tools used, including macular thickness assessment equipment and $\mathrm{ADHD}$ rating scales. Further studies are needed to gain more precise knowledge on this topic. ${ }^{15}$

In children with ADHD, the frontal cortex was thicker than the parietal cortex in the present study. However, there was no difference in thickness between the frontal and parietal corti- 
Table 3. Group differences in the ratios of the frontal-parietal and frontal-temporal cortices

\begin{tabular}{lccccc}
\hline \multirow{2}{*}{ Region } & \multicolumn{2}{c}{ Mean (SD) } & Differences (95\% CI) & p \\
\cline { 2 - 3 } & Controls (N=13) & ADHD (N=12) & & \\
\hline Frontal-parietal (LH) & $115.9(3.7)$ & $116.1(5.0)$ & & $-0.12(-3.74$ to 3.50$)$ & 0.94 \\
Frontal-parietal (RH) & $112.2(2.2)$ & $114.7(3.3)$ & & $-2.47(-4.78$ to -0.16$)$ & 0.04 \\
Frontal-temporal (LH) & $95.0(2.5)$ & $96.3(3.1)$ & & $-1.32(-3.65$ to 1.01$)$ & 0.25 \\
Frontal-temporal (RH) & $92.6(3.0)$ & $94.6(2.5)$ & & $-2.09(-4.37$ to 0.19$)$ & 0.07 \\
\hline
\end{tabular}

The independent t-test was used for group comparisons. The ratios were calculated by (the mean cortical thickness of frontal lobe/the mean cortical thickness of parietal or temporal cortices) $\times 100$. ADHD: attention deficit hyperactivity disorder, LH: left hemisphere, RH: right hemisphere, SD: standard deviation, CI: confidence interval

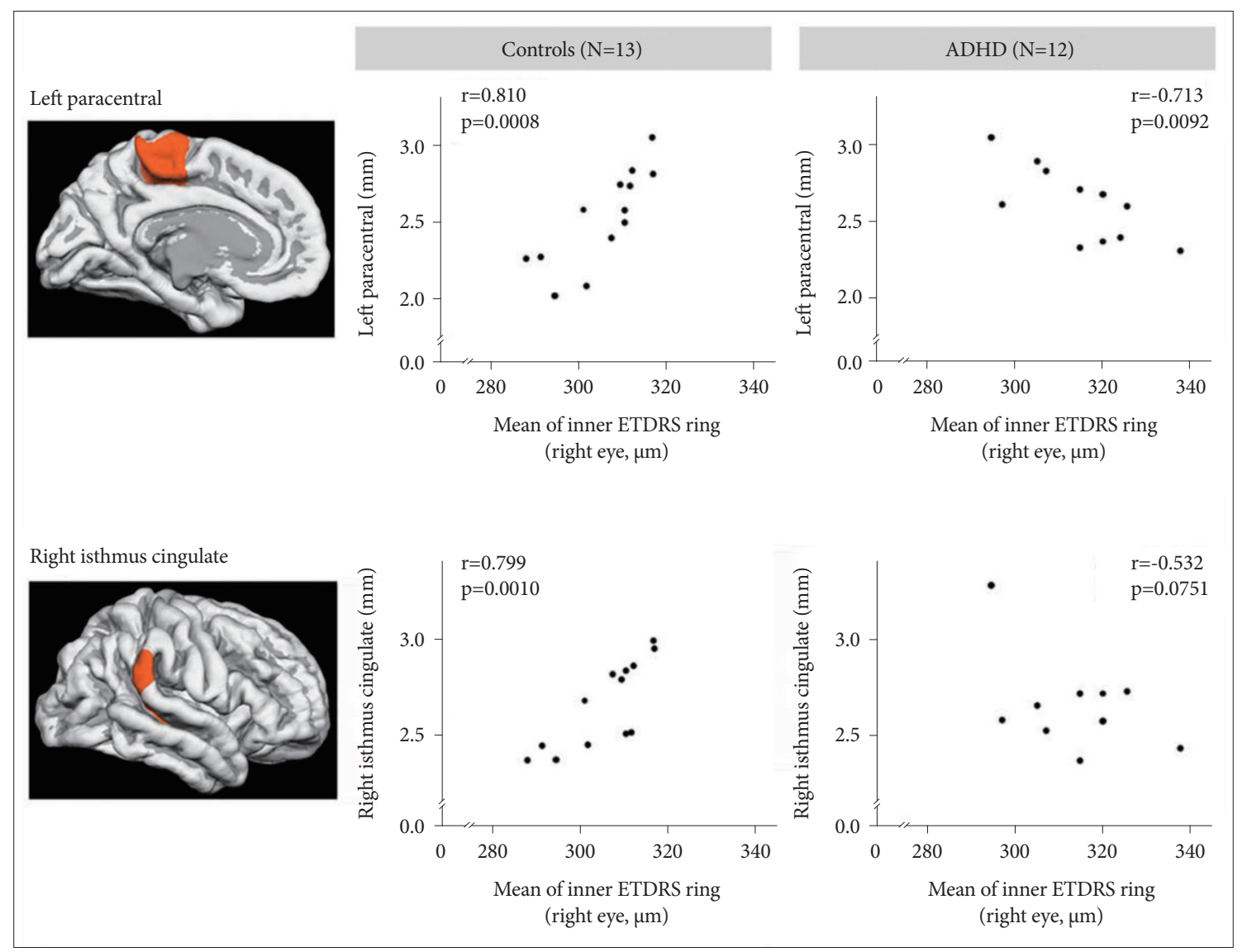

Figure 3. Relationship between macular and cortical thickness in the control group. Pearson correlation test was used for the analysis. All levels of significance were set at $0.0015(0.05 / 34)$, considering 34 regions derived from the Desikan-Killiany atlas to address multiple comparison issues. ETDRS: Early Treatment Diabetic Retinopathy Study, ADHD: attention deficit hyperactivity disorder.

ces. A thick prefrontal cortex (not a reduced cortex volume) in the developing brain of children is thought to be associated with dysfunctional pruning during development. ${ }^{32}$ Longitudinal brain imaging studies of synaptic elimination have suggested that the process of pruning is related to the development of cognitive ability in humans. ${ }^{33}$ The core roles of the frontal cortex are organization, control, and association of relevant information ${ }^{34}$ and the main function of the parietal cortex is to receive information from sensory stimuli from almost all parts of the body. ${ }^{34}$ Deficits within the fronto-parietal circuit have been reported to be associated with attention and impulse control deficits. ${ }^{35,36}$ Considering the results of this study, an imbalance in the fronto-parietal cortex may be associated with the clinical symptoms of ADHD. Several studies have found decreased cortical thickness in the frontal cortex of ADHD groups compared to healthy groups. ${ }^{6,737}$ However, we found no difference in the frontal regions between ADHD and comparison groups. Two factors, namely small sample size and different analysis methods, may account for differences in results between previous studies and ours. Previous studies simply compared ADHD and healthy groups using a whole brain analysis approach; ${ }^{4,6}$ however, we compared the ADHD and 


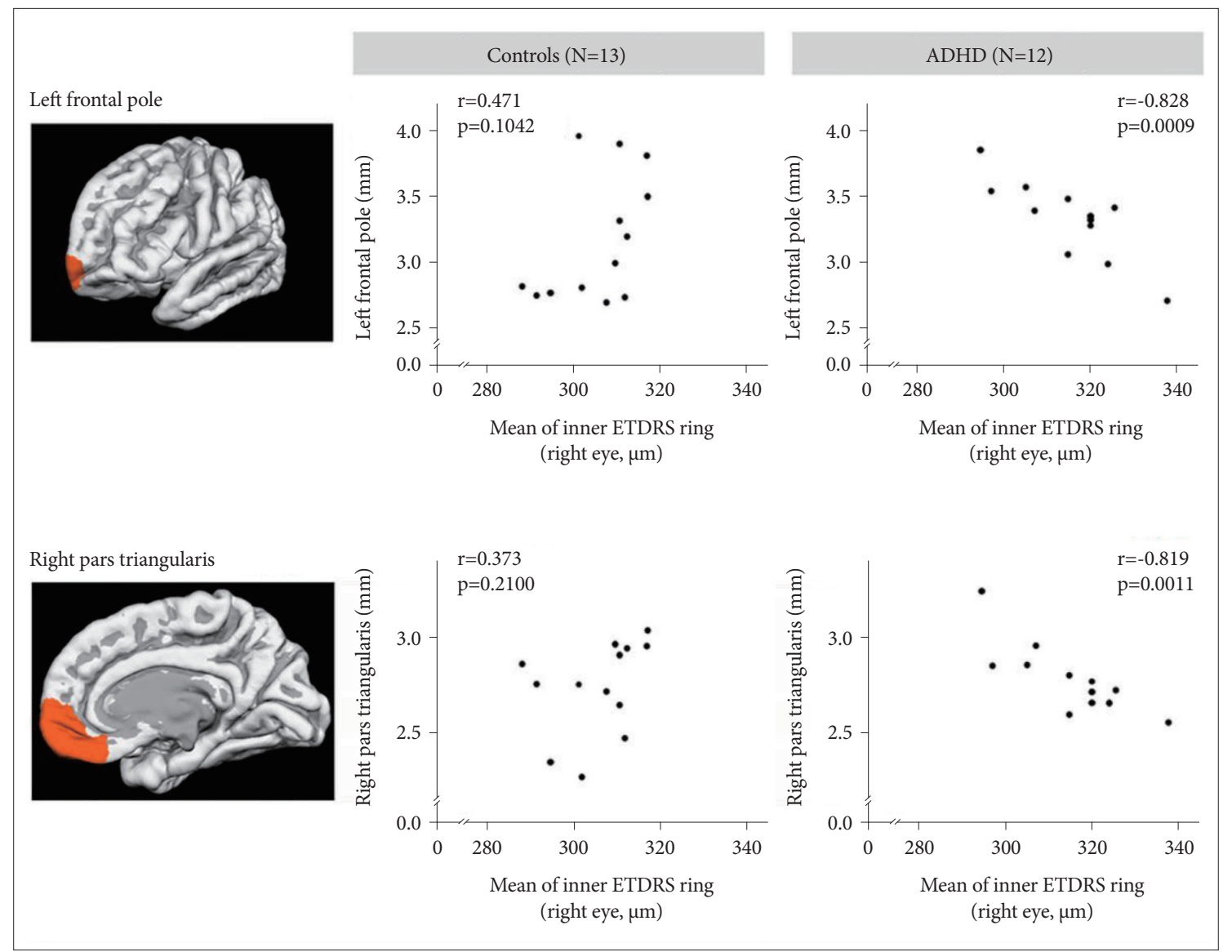

Figure 4. Relationship between macular and cortical thickness in the ADHD group. Pearson correlation test was used for the analysis. All levels of significance were set at $0.0015(0.05 / 34)$, considering 34 regions derived from the Desikan-Killiany atlas to address multiple comparison issues. ETDRS: Early Treatment Diabetic Retinopathy Study, ADHD: attention deficit hyperactivity disorder.

comparison groups using the ratios of the four cortices. We believe that our methodological procedure could provide new insights into brain abnormalities in youth with ADHD.

The eye is derived from the neuroepithelium and the surface ectoderm and shares a common origin with the brain from an embryological perspective. As retinal abnormalities have been used as a candidate surrogate marker for schizophrenia and $\mathrm{AD},{ }^{13,14}$ the outcome of our work suggests that this may also be true for detecting ADHD. Moreover, macular and RNFL thinning in AD has been considered a result of the loss of neurons and ganglion cell axons. ${ }^{38}$ These ideas suggest that our results reflect a parallel between retinal development in children and synaptic pruning in the brain. Brain scans demand excessive time and expenses to conduct such studies, which would present a barrier to neuroimaging research. The concept of retinal measurement as a new marker of brain abnormality may assist in the diagnosis of ADHD.

We found a relationship between macular and cortical thickness in the frontal cortex of children with ADHD for the first time. These compelling results can strengthen the basis for the importance of macular thickness abnormalities as a mark- er in children with ADHD. Our study makes the first step toward demonstrating an association between the retina and the brain, which have the same root embryologically. Qiu et al. ${ }^{39}$ found decreased cortical thickness in the right cingulate cortex in participants with ADHD and decreased functional connectivity in the anterior/posterior cingulate cortices. Interestingly, in the bilateral posterior medial frontal cortex, increased functional connectivity was observed in Qiu et al.s study. These results indicate a trend similar to our findings in correlation analyses regarding right isthmus cingulate thickness in the control group and left frontal pole/right pars triangularis thickness in the ADHD group. Another study with 43 children showed that the control group had a higher cortical thickness in the right posterior cingulate, whereas the ADHD group had an increased cortical thickness in the right pars triangularis. ${ }^{40}$ Results from this study corroborate our aforementioned findings. Overall, previous articles have reported that control groups had higher cortical thickness or functional connectivity in the cingulate region, while ADHD groups had increased cortical thickness or functional connectivity in the posterior medial frontal region. Our findings also demonstrate 
a significant correlation between macular and cortical thickness in these regions, indicating that retinal measurement could be important in detecting abnormal cortical development in a convenient manner.

Our study has several limitations. First, small sample sizes necessitate that we could not generalize our results to the general population. Second, we could not consider relationships between clinical measures and cortical or macular thickness due to our small sample size. Finally, some of our results from this study did not survive after Bonferroni correction.

Our results suggest that the different patterns of macular thickness might represent the immature cortical thickness of brains of children with ADHD. To our knowledge, this is the first investigation to examine the relationship between brain abnormalities and macular thickness in ADHD. More research on potential clinical markers of ADHD will help us gain a better understanding of the ADHD brain, particularly via a longitudinal study design or resting-state functional MRI datasets.

\section{Acknowledgments}

This study was supported by grants from the Korean Health Technology R \&D Project, the Ministry of Health \& Welfare, Republic of Korea (A120013).

\section{Conflicts of Interest}

The authors have no potential conflicts of interest to disclose.

\section{Author Contributions}

Conceptualization: Jee Taek Kim, Doug Hyun Han. Data curation: Jee Taek Kim, Doug Hyun Han. Formal analysis: Sujin Bae, Doug Hyun Han. Funding: Doug Hyun Han. Investigation: Jee Taek Kim, Doug Hyun Han. Methodology: Sujin Bae, Jung Min Han. Supervision: Doug Hyun Han. Visualization: Sujin Bae, Jung Min Han. Writing_original draft: Sujin Bae, Doug Hyun Han. Writing — review \& editing: Sujin Bae, Jee Taek Kim, Jung Min Han, Doug Hyun Han.

\section{ORCID iDs}

Doug Hyun Han https://orcid.org/0000-0002-8314-0767

Sujin Bae https://orcid.org/0000-0002-9671-4627

\section{REFERENCES}

1. Simon V, Czobor P, Balint S, Meszaros A, Bitter I. Prevalence and correlates of adult attention-deficit hyperactivity disorder: meta-analysis. Br J Psychiatry 2009;194:204-211.

2. Thomas R, Sanders S, Doust J, Beller E, Glasziou P. Prevalence of attention-deficit/hyperactivity disorder: a systematic review and meta-analysis. Pediatrics 2015;135:e994-e1001.

3. Kiely B, Adesman A. What we do not know about ADHD... yet. Curr Opin Pediatr 2015;27:395-404.

4. Shaw P, Lerch J, Greenstein D, Sharp W, Clasen L, Evans A, et al. Longitudinal mapping of cortical thickness and clinical outcome in children and adolescents with attention-deficit/hyperactivity disorder. Arch Gen Psychiatry 2006;63:540-549.

5. Vaidya CJ. Neurodevelopmental abnormalities in ADHD. Curr Top Behav Neurosci 2012;9:49-66.

6. Almeida Montes LG, Prado Alcantara H, Martinez Garcia RB, De La Torre LB, Avila Acosta D, Duarte MG. Brain cortical thickness in ADHD: age, sex, and clinical correlations. J Atten Disord 2013;17:641-654.
7. Hoekzema E, Carmona S, Ramos-Quiroga JA, Richarte Fernandez V, Picado M, Bosch R, et al. Laminar thickness alterations in the frontoparietal cortical mantle of patients with attention-deficit/hyperactivity disorder. PLoS One 2012; 7:e48286.

8. Fernandez-Jaen A, Lopez-Martin S, Albert J, Fernandez-Mayoralas DM, Fernandez-Perrone AL, Tapia DQ, et al. Cortical thinning of temporal pole and orbitofrontal cortex in medication-naive children and adolescents with ADHD. Psychiatry Res 2014;224:8-13.

9. Shaw P, Lalonde F, Lepage C, Rabin C, Eckstrand K, Sharp W, et al. Development of cortical asymmetry in typically developing children and its disruption in attention-deficit/hyperactivity disorder. Arch Gen Psychiatry 2009;66:888-896.

10. Hunt RH, Thomas KM. Magnetic resonance imaging methods in developmental science: a primer. Dev Psychopathol 2008;20:1029-1051.

11. MacCormick IJ, Czanner G, Faragher B. Developing retinal biomarkers of neurological disease: an analytical perspective. Biomark Med 2015; 9:691-701.

12. Ascaso FJ, Rodriguez-Jimenez R, Cabezon L, Lopez-Anton R, Santabarbara J, De la Camara C, et al. Retinal nerve fiber layer and macular thickness in patients with schizophrenia: Influence of recent illness episodes. Psychiatry Res 2015;229:230-236.

13. Kirbas S, Turkyilmaz K, Anlar O, Tufekci A, Durmus M. Retinal nerve fiber layer thickness in patients with Alzheimer disease. J Neuroophthalmol 2013;33:58-61.

14. Iseri PK, Altinas O, Tokay T, Yuksel N. Relationship between cognitive impairment and retinal morphological and visual functional abnormalities in Alzheimer disease. J Neuroophthalmol 2006;26:18-24.

15. Herguner A, Alpfidan I, Yar A, Erdogan E, Metin O, Sakarya Y, et al. Retinal nerve fiber layer thickness in children with ADHD. J Atten Disord 2016; 22:619-626.

16. Kim YS, Cheon KA, Kim BN, Chang SA, Yoo HJ, Kim JW, et al. The reliability and validity of Kiddie-Schedule for Affective Disorders and Schizophrenia-Present and Lifetime Version- Korean version (K-SADSPL-K). Yonsei Med J 2004;45:81-89.

17. Kovacs M. The Children's Depression, Inventory (CDI). Psychopharmacol Bull 1985;21:995-998.

18. So YK, Noh JS, Kim YS, Ko SG, Koh YJ. The reliability and validity of Korean Parent and Teacher ADHD Rating Scale. J Korean Nueropsychiatr Assoc 2002;41:283-289.

19. Focal photocoagulation treatment of diabetic macular edema. Relationship of treatment effect to fluorescein angiographic and other retinal characteristics at baseline: ETDRS report no. 19. Early Treatment Diabetic Retinopathy Study Research Group. Arch Ophthalmol 1995;113: 1144-1155.

20. Reuter M, Rosas HD, Fischl B. Highly accurate inverse consistent registration: a robust approach. Neuroimage 2010;53:1181-1196.

21. Segonne F, Dale AM, Busa E, Glessner M, Salat D, Hahn HK, et al. A hybrid approach to the skull stripping problem in MRI. Neuroimage 2004;22:1060-1075.

22. Fischl B, Salat DH, Busa E, Albert M, Dieterich M, Haselgrove C, et al. Whole brain segmentation: automated labeling of neuroanatomical structures in the human brain. Neuron 2002;33:341-355.

23. Sled JG, Zijdenbos AP, Evans AC. A nonparametric method for automatic correction of intensity nonuniformity in MRI data. IEEE Trans Med Imaging 1998;17:87-97.

24. Fischl B, Liu A, Dale AM. Automated manifold surgery: constructing geometrically accurate and topologically correct models of the human cerebral cortex. IEEE Trans Med Imaging 2001;20:70-80.

25. Dale AM, Fischl B, Sereno MI. Cortical surface-based analysis. I. Segmentation and surface reconstruction. Neuroimage 1999;9:179-194.

26. Fischl B, Dale AM. Measuring the thickness of the human cerebral cortex from magnetic resonance images. Proc Natl Acad Sci U S A 2000; 97:11050-11055.

27. Fischl B, Sereno MI, Dale AM. Cortical surface-based analysis. II: Inflation, flattening, and a surface-based coordinate system. Neuroimage 
1999;9:195-207.

28. Fischl B, Sereno MI, Tootell RB, Dale AM. High-resolution intersubject averaging and a coordinate system for the cortical surface. Hum Brain Mapp 1999;8:272-284.

29. Desikan RS, Segonne F, Fischl B, Quinn BT, Dickerson BC, Blacker D, et al. An automated labeling system for subdividing the human cerebral cortex on MRI scans into gyral based regions of interest. Neuroimage 2006;31:968-980.

30. Cardinale F, Chinnici G, Bramerio M, Mai R, Sartori I, Cossu M, et al. Validation of FreeSurfer-estimated brain cortical thickness: comparison with histologic measurements. Neuroinformatics 2014;12:535-542.

31. Kuperberg GR, Broome MR, McGuire PK, David AS, Eddy M, Ozawa F, et al. Regionally localized thinning of the cerebral cortex in schizophrenia. Arch Gen Psychiatry 2003;60:878-888.

32. Sowell ER, Mattson SN, Kan E, Thompson PM, Riley EP, Toga AW. Abnormal cortical thickness and brain-behavior correlation patterns in individuals with heavy prenatal alcohol exposure. Cereb Cortex 2008;18: 136-144.

33. Blakemore SJ, Choudhury S. Development of the adolescent brain: implications for executive function and social cognition. J Child Psychol
Psychiatry 2006;47:296-312.

34. Gazzaniga MS, Ivry RB, Mangun GR. Cognitive Neuroscience: The Biology of the Mind. New York: W. W. Norton \& Company; 2014.

35. Corbetta M, Shulman GL. Control of goal-directed and stimulus-driven attention in the brain. Nat Rev Neurosci 2002;3:201-215.

36. Corbetta M, Kincade MJ, Lewis C, Snyder AZ, Sapir A. Neural basis and recovery of spatial attention deficits in spatial neglect. Nat Neurosci 2005;8:1603-1610

37. Shaw P, Eckstrand K, Sharp W, Blumenthal J, Lerch JP, Greenstein D, et al. Attention-deficit/hyperactivity disorder is characterized by a delay in cortical maturation. Proc Natl Acad Sci U S A 2007;104:19649-19654.

38. Guo L, Duggan J, Cordeiro MF. Alzheimer's disease and retinal neurodegeneration. Curr Alzheimer Res 2010;7:3-14.

39. Qiu MG, Ye Z, Li QY, Liu GJ, Xie B, Wang J. Changes of brain structure and function in ADHD children. Brain Topogr 2011;24:243-252.

40. Almeida LG, Ricardo-Garcell J, Prado H, Barajas L, Fernandez-Bouzas A, Avila D, et al. Reduced right frontal cortical thickness in children, adolescents and adults with ADHD and its correlation to clinical variables: a cross-sectional study. J Psychiatr Res 2010;44:1214-1223. 